\title{
Effect of Antibiotic Treatment on the Gastrointestinal Microbiome of Free- Ranging Western Lowland Gorillas (Gorilla g. gorilla)
}

\begin{abstract}
Authors: Klára Vlčková, Andres Gomez, Klára J. Petrželková, Christopher A. Whittier, Angelique F. Todd, Carl J. Yeoman, Karen E. Nelson, Brenda A. Wilson, Rebecca M. Stumpf, David Modrý, Bryan A. White, and Steven R. Leigh
\end{abstract}

The final publication is available at Springer via http://dx.doi.org/10.1007/s00248-016-0745-5.

Vlckova, Klara, Andres Gomez, Klara J Petrzelkova, Christopher A Whittier, Angelique F Todd, Carl J Yeoman, Karen E Nelson, Brenda A Wilson, Rebecca M Stumpf, David Modry, Bryan A White, and Steven R Leigh. "Effect of Antibiotic Treatment on the Gastrointestinal Microbiome of Free-Ranging Western Lowland Gorillas (Gorilla g. gorilla)." Microbial Ecology 72, no. 4 (November 2016): 943-954. DOI: 10.1007/s00248-016-0745-5. 


\title{
Effect of Antibiotic Treatment on the Gastrointestinal Microbiome of Free-Ranging Western Lowland Gorillas (Gorilla g. gorilla)
}

\author{
Klára Vlčková $^{1}$ • Andres Gomez ${ }^{2,3}$ • Klára J. Petrželková ${ }^{4,5,6}$ • \\ Christopher A. Whittier ${ }^{7,8}$ - Angelique F. Todd ${ }^{9}$ - Carl J. Yeoman ${ }^{10}$. \\ Karen E. Nelson ${ }^{11,16}$ • Brenda A. Wilson ${ }^{3,12}$ • Rebecca M. Stumpf ${ }^{3,13}$. \\ David Modrý ${ }^{1,6,14}$ • Bryan A. White ${ }^{3}$ Steven R. Leigh ${ }^{3,15}$
}

1 Department of Pathology and Parasitology, Faculty of Veterinary Medicine, University of Veterinary and Pharmaceutical Sciences Brno, Palackého tř. 1/3, Brno 61242, Czech Republic Jolla, CA 92037, USA

3 Carl R. Woese Institute for Genomic Biology, University of Illinois at Urbana-Champaign, 1206 West Gregory Drive, Urbana, IL 61801, USA Institute of Vertebrate Biology, Academy of Sciences of the Czech Republic, Květná 8, Brno 60365, Czech Republic Liberec Zoo, Masarykova 1347/31, Liberec 46001, Czech Republic

6 Institute of Parasitology, Biology Centre of the Academy of Sciences of the Czech Republic, Branišovská 31, České Budějovice 37005, Czech Republic

7 Cummings School of Veterinary Medicine at Tufts University, 200 Westboro Road, North Grafton, MA 01536, USA

8 Department of Wildlife Health Sciences, Smithsonian Conservation Biology Institute, National Zoological Park, 3001 Connecticut Avenue Northwest, Washington, DC 20008, USA

9 WWF, Dzanga Sangha Protected Areas, BP 1053, Bangui, Central African Republic
10 Department of Animal and Range Sciences, Montana State University, P.O. Box 172900, Bozeman, MT 59717-2900, USA

11 J. Craig Venter Institute, 9704 Medical Center Drive, Rockville, MD 20850, USA

12 Department of Microbiology, University of Illinois at Urbana-Champaign, 601 South Goodwin Avenue, Urbana, IL 61801, USA

13 Department of Anthropology, University of Illinois at Urbana-Champaign, 607 South Mathews Avenue, Urbana, IL 61801, USA

14 CEITEC VFU, University of Veterinary and Pharmaceutical Sciences Brno, Palackého tř. 1/3, Brno 61242, Czech Republic

15 Department of Anthropology, University of Colorado at Boulder, 1350 Pleasant Street, Boulder, CO 80309-0233, USA

16 J. Craig Venter Institute, 4120 Capricorn Lane, La Jolla, CA 92037 , USA

\begin{abstract}
The mammalian gastrointestinal (GI) microbiome, which plays indispensable roles in host nutrition and health, is affected by numerous intrinsic and extrinsic factors. Among them, antibiotic (ATB) treatment is reported to have a signif-icant effect on GI microbiome composition in humans and other animals. However, the impact of ATBs on the GI microbiome of free-ranging or even captive great apes remains poorly characterized. Here, we investigated the effect of cephalosporin treatment (delivered by intramuscular dart injection during a serious respiratory outbreak) on the GI microbiome of a wild habituated group of western lowland gorillas (Gorilla gorilla gorilla) in the Dzanga Sangha Protected Areas, Central African Republic. We examined 36 fecal samples from eight individuals, including samples be-fore and after ATB treatment, and characterized the GI microbiome composition using Illumina-MiSeq sequencing of the bacterial 16S rRNA gene. The GI microbial profiles of samples from the same individuals before and after ATB administration indicate that the ATB treatment impacts GI microbiome stability and the relative abundance of particular bacterial taxa within the colonic ecosystem of wild gorillas. We observed a statistically significant increase in Firmicutes and a decrease in Bacteroidetes levels after ATB treatment. We found disruption of the fibrolytic community linked with adecreaseofRuminoccocus levels as a result of ATB treatment. Nevertheless, the nature of the changes observed after ATB treatment differs among gorillas and thus is dependent on the individual host. This study has important implications for ecology, management, and conservation of wild primates.
\end{abstract}




\section{Introduction}

The mammalian gastrointestinal (GI) microbiome is a complex system, with vital physiological and immunological roles. Production of vitamins and fermentation of non-digestible dietary components underscore the contribution of the GI microbiome to host metabolism and nutrition [1]. Similarly, the GI microbiome is an integral part of the host's immune system, protecting the host from infection by pathogenic microorganisms and constituting a mucosal defense barrier $[1,2]$.

It is widely known that administration of antimicrobial agents has significant effects on the composition and activity of GI microbiome in humans and laboratory animals [3-8]. Antibiotic (ATB) treatment is used to suppress bacterial infections in humans and animals; however, it usually results in a decrease in the diversity of the gut microbiome, changes in microbial composition, and dysregulation of the host's immune homeostasis, causing higher susceptibility to other diseases [4, 9], as well as emergence of ATB-resistant strains [10, 11]. While the various ATBs target specific bacterial groups, non-target microbes may also be differentially impacted by ATB treatment [12] due to their exchanges of secondary metabolites or cross-feed products with the targeted species [9]. Even short-term administration of ATBs can result in longterm changes in the climax structure of the fecal microbiome of humans, altering the composition and relative abundance of specific taxa $[4,12]$.

Several studies have examined the impact of ATBs on the GI microbiome of various hosts, e.g., humans [4, 6-8], swine [13], and mice [14]. However, the impact of ATBs on the GI microbiome of non-human great apes remains unknown, despite the fact that various ATBs are often used in captive and sometimes even free-ranging great apes to treat various infections. For instance, wild mountain gorillas (Gorilla beringei beringei) from Volcanoes National Park in Rwanda have been treated with ATBs including ceftriaxone for respiratory diseases $[15,16]$. Cefovecin has been used for treating respiratory outbreaks in captive chimpanzees (Pan troglodytes), western lowland gorillas (Gorilla gorilla gorilla), and Bornean orangutans (Pongo pygmaeus) [17].
Here, we opportunistically studied the effect of ATB treatment on a fully habituated group of wild western lowland gorillas in Dzanga Sangha Protected Areas (DSPA), Central African Republic, during a serious respiratory disease outbreak that occurred on February to April 2012. EXCEDE $^{\circledR}$ (ceftiofur crystalline-free acid) Sterile Suspension and Ceftriaxone MYLAN ${ }^{\circledR}$ were the ATBs used to treat the gorillas in this study. Both are third-generation cephalosporin formulations with a broad spectrum activity against grampositive and gram-negative bacteria, including B-lactamaseproducing strains (Zoetis Inc., Madison, WI, USA; MYLAN $^{\circledR}$, Belgium). Like other cephalosporins, ceftiofur is bactericidal, via disruption of cell wall synthesis, which occurs in the peptidoglycan layer of the bacterial cell wall that maintains cell rigidity. B-lactam agents bind to penicillinbinding proteins (essential for synthesis of the bacterial wall), inhibiting cell wall synthesis and ultimately causing cell lysis [18]. EXCEDE is used for intramuscular administration in horses and other domestic species [19, 20]. Nevertheless, due to its prolonged duration of action, it is also increasingly used in wildlife species requiring less frequent injections to minimize handling and stress [21,22]. The ATB treatment was administrated because of the highly productive (i.e., audible expectoration) nature of the coughs, which differed markedly from four previously observed respiratory outbreaks 
in DSPA-habituated gorilla groups, the prolonged history of symptoms in some individuals, and the observed spread of symptoms within the group. Moreover, there was a risk of a bacterial infection, possibly initiated by a viral infection, which, based on experience in mountain gorillas, may result in the sudden death of asymptomatic juveniles and infants [16]. The fully habituated status of the lowland gorillas allowed monitoring and collection of fecal samples from individuals before and after ATB administration, as well as subsequent assessment of the overall impact of ATB on the composition of GI microbiome.

\section{Material and Methods}

\section{Study Site and Sample Collection}

The study was conducted in the Dzanga sector of the DzangaNdoki National Park (part of the DSPA Complex), Central African Republic (Fig. S1). Fecal samples from eight individual gorillas from the fully habituated group Makumba (Table S1) were collected in the surroundings of the Bai Hokou research camp $\left(2^{\circ} 50^{\prime} \mathrm{N}, 16^{\circ} 28^{\prime}\right.$ E) from February to April 2012. The time of fecal sample collection and date of ATB application in each individual are listed in Table 1. Thirty-two fecal samples composed of 13 samples collected before ATB treatment and 19 samples after ATB treatment were obtained. Four samples from two gorillas that were not subjects of ATB treatment were also collected. ATB treatment was given to the gorillas between March 10 and 13, 2012. Ceftriaxone ( $1 \mathrm{~g}$, IM; MYLAN ${ }^{\circledR}$, Belgium) was used for the first treatment dose of a gorilla named Mossoko, and subsequent treatments were with EXCEDE $^{\circledR}(\sim 1.4 \mathrm{mg} \mathrm{CE} / \mathrm{kg}, \mathrm{IM}$, ceftiofur crystalline-free acid; Zoetis Inc., Madison, USA) for all other treated gorillas including Mossoko (for the second dose). Fecal samples were obtained non-invasively, immediately after defecation during gorilla follows. Samples (approximately $0.2 \mathrm{~g}$ of feces) were mixed with $1.5 \mathrm{ml}$ of RNAlater (QIAGEN, CA, USA) and kept at room temperature for a maximum of 1 month before transport to the Department of Pathology and Parasitology, University of Veterinary and Pharmaceutical Sciences Brno, Czech Republic, where they were stored at $-80{ }^{\circ} \mathrm{C}$. Samples were shipped to the Institute for Genomic Biology, University of Illinois at UrbanaChampaign, USA, for DNA sequencing analyses in November 2012. The non-invasive sample collection was conducted according to the Convention on Human Rights and Biomedicine of the Council of Europe and Directive 86/ 609/EEC on the Protection of Animals Used for Experimental and Other Scientific Purposes and meet the International Guiding Principles for Biomedical Research involving Animals, as issued by the Council for International Organizations of Medical Sciences (C.I.O.M.S., c/o WHO,
CH 1211 Geneva 27, Switzerland). The research adhered to the legal requirements of the Central African Republic. Importation of the samples to the EU was approved by the State Veterinary Authority of the Czech Republic. The sequencing was conducted under University of Illinois IACUC protocol 11046.

\section{Sample Processing and Sequencing Analyses}

DNA from fecal samples was extracted using the Ultra Clean Soil DNA Isolation Kit (MoBio Laboratories Inc., Carlsbad, CA, USA) according to the manufacturer's protocol. DNA was purified using QIAquick Gel Extraction Kit (QIAGEN, Germany). The V3 and V5 regions of $16 \mathrm{~S}$ ribosomal ribonucleic acid (rRNA) were amplified by polymerase chain reaction $\left(25\right.$ cycles of $98^{\circ} \mathrm{C}(15 \mathrm{~s}), 65^{\circ} \mathrm{C}(30 \mathrm{~s})$, and $\left.72^{\circ} \mathrm{C}(30 \mathrm{~s})\right)$ using the primer set $357 \mathrm{f}\left(5^{\prime}\right.$-CCTACGGGAGGCAGCAG$\left.3^{\prime}\right)$ and 926r (5'-CCGTCAATTCMTTTRAGT-3') tagged with identifying bar codes (Table S2). Because some of the obtained PCR amplicons were of low DNA concentration, we performed a second round of amplification on these amplicons, using the same primer set and PCR conditions. To determine the effect of the second round of amplification, we performed the same procedure in all amplicons, including those with strong DNA concentration. Amplicon purification was done using the QIAquick PCR Purification Kit (QIAGEN, Germany). Amplicon sequencing was performed using the Illumina MiSeq Platform at the Roy J. Carver Biotechnology Center, University of Illinois at UrbanaChampaign (Urbana, IL). Samples with $<4000$ reads after Illumina sequencing were removed from all subsequent analyses to avoid biases in diversity estimations. The reads were clustered into operational taxonomic units (OTUs) using the online tool IM-TORNADO as described in Jeraldo et al. [23]. Briefly, reads were filtered for quality, and non-overlapping pair-end reads were joined, concatenated, and sorted by cluster to find de novo OTUs using USEARCH and its UPARSE algorithm, which also removed chimeric sequences. Taxonomy assignments were carried out using Ribosomal Database Project's naive Bayesian classifier against the Greengenes 13_5 database. Fully unclassified reads were then eliminated. After taxonomy assignment, reads were aligned and mapped against their corresponding set of unaligned. OTU representatives were defined using USEARCH at $97 \%$ sequence identity to generate an OTU table. OTUs detected less than three times in a sample and/or occurring less than five reads in the entire data set were discarded to avoid including probable sequence artifacts in the analyses.

\section{Statistical Analyses}

Relative abundance of each OTU and Bray-Curtis dissimilarity matrices were used for principal coordinate (PCO) 
Table 1 Timetable of samples of individual gorillas during respiratory outbreak

\begin{tabular}{|c|c|c|c|c|c|c|c|c|c|c|c|c|c|c|c|c|c|c|c|c|c|c|c|c|c|c|c|c|c|c|c|c|c|c|c|}
\hline Individual/ & \multicolumn{9}{|c|}{ February } & \multicolumn{17}{|c|}{ March } & \multicolumn{9}{|c|}{ April } \\
\hline Date & 17 & 22 & 23 & 242 & 25 & 26 & 27 & 28 & 29 & 1 & 2 & 3 & 4 & 5 & 6 & 7 & 8 & \begin{tabular}{l|l}
9 & 1 \\
\end{tabular} & 10 ? & 11 & 12 & 13 & 14 & 15 & 24 & 25 & 2 & 3 & & 14 ] 1 & \begin{tabular}{l|l}
15 & 1 \\
\end{tabular} & \begin{tabular}{l|l}
16 & 1 \\
\end{tabular} & 192 & & 24 \\
\hline Infection day & 1 & 6 & 7 & 8 & 9 & 10 & 11 & 12 & 13 & 14 & 15 & 16 & 17 & 18 & 19 & 202 & 212 & 222 & 232 & 24 & 25 & 26 & & & & & & & & & & & & & \\
\hline Makumba & & & & $x$ & $\mathrm{x}$ & & & & & & & $\mathrm{x}$ & & $\mathrm{x}$ & & $\mathrm{x}$ & & & & & $\mathrm{x}$ & & & $x$ & $\mathrm{x}$ & $x$ & & & $x$ & $x$ & $x$ & $\mathrm{x}$ & & & \\
\hline Malui & & & & & & & & $\mathrm{x}$ & & & & & & & & & & & & & $\mathrm{x}$ & & & $x$ & $\mathrm{x}$ & & & & & $x$ & & & & & \\
\hline Mopambi & & & & & & & & & & & & & & & & & & & & & $x$ & & $\mathrm{x}$ & $x$ & & & & & & & & & & & \\
\hline Mossoko & & & & & & & & & & & & & & & & & & & $\mathrm{x}$ & $\mathrm{x}$ & $x$ & & & & & & & & & & & & & & \\
\hline Tembo & & & & $\mathrm{x}$ & & & & & & & & & & & & & & & & & & & & & & & & & & & $\mathrm{x}$ & & & & \\
\hline Mobangui & & & & $\mathrm{x}$ & & & $\mathrm{x}$ & & & & & & & & & & & & & & & & & $x$ & & $x$ & & $x$ & & & & & & & \\
\hline Sopo & & & & & & & & & & & & & & & & & & & & & $\mathrm{x}$ & & & & & & & & & & & & & & \\
\hline Kunga & & & & & & & & & & & & & & $x$ & & & & & & & & & & & & & & & & $x$ & $\mathrm{x}$ & & & & \\
\hline
\end{tabular}

Dark grey day of ATB treatment, pink days after ATB treatment, $x$ collection of sample. Detailed information about samples are provided in Table S1

analyses and analysis of similarities (ANOSIM) in PRIMER6 for Windows v.6.1.10 [24]. The stacked bar plots of microbial profiles were generated with QIIME v.1.7.0 [25]. Taxa characterizing samples from before and after ATB treatment were identified based on the frequency and relative abundance of taxa using indicator species analysis [26] in the labdsv R package $[27,28]$. These analyses were performed at the microbial genus, family, and phylum levels for all samples collected before and after ATB treatment and also individually for the one individual with more than four samples collected before and after ATB treatment (the silverback male, Makumba). The vegan package of R [29] was used for the analysis of multivariate homogeneity of group dispersions, rarefied richness analysis, calculation of Shannon diversity index, and permutational analysis of variance (PERMANOVA). Boxplots, $t$ tests, Wilcoxon's rank sum tests, and one-way ANOVAs were calculated using stats package in $\mathrm{R}$ [28].

\section{Results}

We validated the two-round PCR approach with analyses of a subset of samples amplified by both a one-round and tworound PCR approach. We observed no statistically significant differences between the GI microbial profiles from 16S rRNA amplicons obtained using either one-round PCR or two-round PCR (Fig. S2) according to ANOSIM $(R=0.005, p=0.378)$ and PERMANOVA (pseudo $F=1.501, p=0.105$ ). We did not detect statistically significant differences in GI bacterial diversity between samples processed by one-round PCR and tworound PCR according to the Shannon index ( $t$ test $t=0.881$, $p=0.386$; Fig. S3) and rarefied richness analysis ( $t$ test $t=0.657, p=0.516$ ). Thus, the set of samples processed by two-round PCR was used for all further analyses. In total, we obtained 16,165,656 high-quality $16 \mathrm{~S}$ rRNA reads with a median sampling depth of 128,645 reads per sample (minimum 34,927 and maximum $1,158,244$ ) after sequence quality control. Non-overlapping pair-end reads yielded a median sequence length of $450 \mathrm{nt}$ ( $250 \mathrm{nt}$ with R1 and $200 \mathrm{nt}$ with R2).
PERMANOVA revealed small but significant differences before and after ATB treatment (pseudo $F=1.968, p=0.021$; Fig. 1). The relative abundance of specific taxa changed after ATB treatment (Fig. S4a), with Firmicutes increasing and Bacteroidetes decreasing in most individuals (Fig. S4a and Table 2). Unclassified members of the Desulfovibrionales and Fibrobacteres increased after ATB treatment. This was also the case for Coriobacterium, while the opposite pattern was seen with Ruminococcus (decreased after ATB treatment; Table 2). We also observed changes in the relative abundance of minor bacterial phyla after ATB treatment; however, these were not statistically significant (Fig. S4b).

There were no significant differences in GI bacterial diversity in samples before and after ATB treatment from all gorillas analyzed collectively according to the Shannon index (ANOVA $F=0.106, p=0.747$ ) and rarefied richness analysis (ANOVA $F=1.093, p=0.306$ ). However, the microbiome of gorillas after ATB treatment was significantly more dispersed and less homogeneous (ANOVA $F=7.86, p=0.009$; Fig. 2). Despite not detecting overall differences in alpha diversity, we found that the impact of ATB treatment on GI microbiome was largely dependent on the individual host. That is, when taking the host into account in the statistic models, individual gorilla was a significant factor explaining Shannon diversity (ANOVA $F=2.282, p=0.077$; Table S3), rarefied richness (ANOVA $F=2.791, p=0.0389$; Table $\mathrm{S} 4$ ), microbial homogeneity (ANOVA $F=4.293, p=0.005$; Table S5), and overall community composition (PERMANOVA pseudo $F=1.782$, $p=0.006$; Table S6) after ATB treatment.

The analysis of samples from the silverback male (Makumba), the individual from which we obtained the highest number of samples before and after ATB treatment (six samples before and six samples after), indicated that bacterial community composition changed significantly after ATB administration (PERMANOVA pseudo $F=1.679$, $p=0.043$ ). The silverback's gut microbiome was significantly more variable (Wilcoxon's rank sum test $W=3, p=0.015$; Fig. 3a) and more dispersed (Fig. 3b) in the samples collected after ATB treatment reflecting the instability of microbiome. We also observed a possible recovery of the silverback's GI 


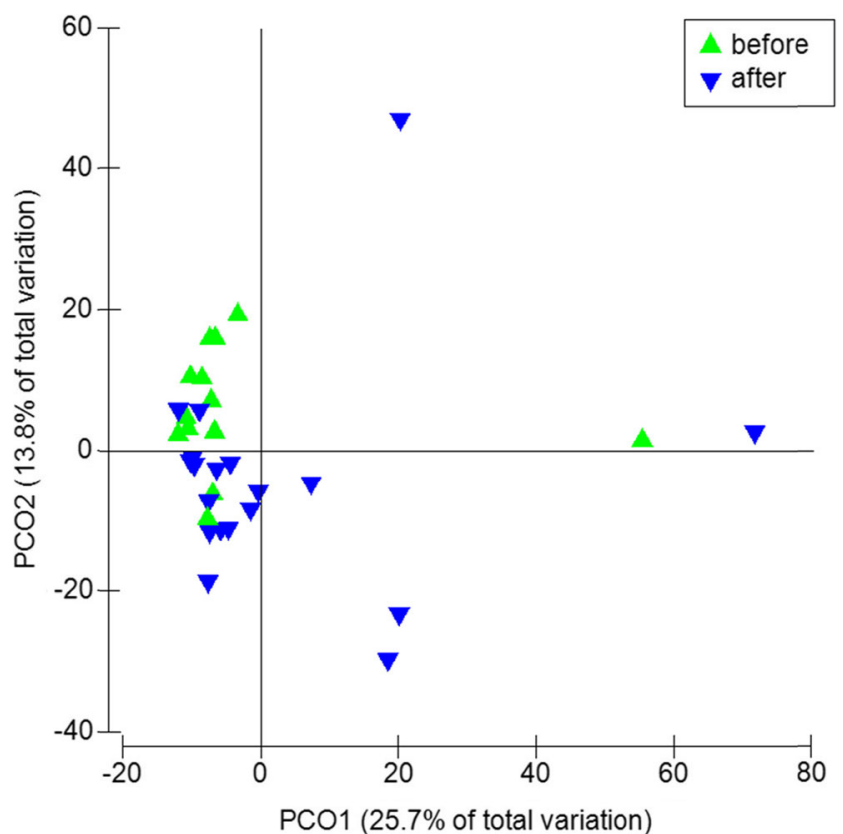

Fig. 1 PCO plot of bacterial community in samples collected before and after ATB treatment

microbiome (Fig. 3a). Microbial profiles in Makumba's samples collected before ATB treatment were very similar to those in samples collected shortly after ATB treatment (from 2 to 12 days after treatment; three samples). However, microbial profiles in Makumba's sample collected 25 days after treatment differed from the others. Microbial profiles in two of his samples collected even later ( 33 and 37 days after treatment) were again similar to those in samples collected before ATB treatment (Fig. 3a). Differences in GI bacterial alpha diversity according to the Shannon index (Wilcoxon's rank sum test $W=32, p=0.026$ ) and rarefied richness analysis (Wilcoxon's rank sum test $W=0, p=0.002$ ) were also significant, indicating that the GI microbiome of the silverback after ATB treatment was less diverse. Analysis of changes in the relative abundance of specific taxa showed that Bacteroidetes decreased significantly in Makumba after ATB treatment (Fig. 4 and Table 3). At the family level, Bacteroidaceae and Spirochaetaceae also decreased in abundance, whereas Anaerolinaceae increased after ATB treatment. At the genus level, Ruminococcus, Lachnobacterium, Sphaerochaeta, and Mycoplasma significantly decreased after ATB administration (Table 3). Similar trends were observed in samples from the remaining gorillas of the group. However, the impact of ATB treatment on the relative abundances of individual taxa varied in each host (Fig. 5).

Microbiome compositions in two individuals that did not receive ATB treatment (the blackback male, Kunga, and the infant, Sopo) were also assessed (Fig. S5). We detected approximately similar levels of all indicator taxa in samples collected during the respiratory outbreak before the ATB treatment of the silverback Makumba and in blackback Kunga. However, differences in levels of indicator taxa were observed specifically in case of Ruminococcus in the period after ATB treatment (Fig. S6).

Table 2 Indicator taxa with mean relative abundances in samples before and after ATB treatment

\begin{tabular}{|c|c|c|c|c|c|}
\hline \multirow[t]{2}{*}{ Taxonomic rank } & \multirow[t]{2}{*}{ Indicator taxa before ATB } & \multirow[t]{2}{*}{ Indicator value } & \multirow[t]{2}{*}{ Probability } & \multicolumn{2}{|c|}{ Mean relative abundance $\%(\mathrm{SD})$} \\
\hline & & & & Before ATB & After ATB \\
\hline Phyla & Bacteroidetes & 0.61 & 0.005 & $39.39 * * *(14.03)$ & $24.69(11.20)$ \\
\hline Family & Unclassified (Mollicutes) & 0.86 & 0.002 & $0.15(0.46)$ & $0.01(0.02)$ \\
\hline Family & Unclassified (Deltaproteobacteria) & 0.47 & 0.027 & $0.02(0.05)$ & $0.01(0.03)$ \\
\hline Genus & Unclassified (Mollicutes) & 0.86 & 0.001 & $0.15(0.46)$ & $0.01(0.02)$ \\
\hline Genus & Ruminococcus & 0.66 & $<0.05$ & $0.43 * *(0.34)$ & $0.22(0.22)$ \\
\hline Genus & Unclassified (Deltaproteobacteria) & 0.47 & 0.01 & $0.02(0.05)$ & $0.01(0.03)$ \\
\hline \multirow[t]{2}{*}{ Taxonomic rank } & \multirow[t]{2}{*}{ Indicator taxa after ATB } & \multirow[t]{2}{*}{ Indicator value } & \multirow[t]{2}{*}{ Probability } & \multicolumn{2}{|c|}{ Mean relative abundance \% (SD) } \\
\hline & & & & Before ATB & After ATB \\
\hline Phyla & Firmicutes & 0.6 & 0.003 & $27.24(4.8)$ & $40.55^{* * *}(14.82)$ \\
\hline Family & Coriobacteriaceae & 0.73 & 0.05 & $2.41(1.81)$ & $8.01(13.01)$ \\
\hline Family & Unclassified (Desulfovibrionales) & 0.57 & 0.045 & $0.01(0.02)$ & $0.06^{*}(0.11)$ \\
\hline Family & Unclassified (Fibrobacteres) & 0.40 & 0.049 & 0 & $0.01 *(0.01)$ \\
\hline Genus & Coriobacterium & 0.64 & $<0.05$ & $0.17(0.07)$ & $0.34 * *(0.27)$ \\
\hline Genus & Unclassified (Desulfovibrionales) & 0.57 & $<0.05$ & $0.01(0.02)$ & $0.06^{*}(0.11)$ \\
\hline Genus & Unclassified (Fibrobacteres) & 0.40 & $<0.05$ & 0 & $0.01 *(0.01)$ \\
\hline
\end{tabular}

Significant differences based on ANOVA, $* * * 0.01, * * 0.05$, and $* 0.1$ 
Fig. 2 GI microbiome dispersion of all gorillas before and after ATB treatment $(p=0.009$, ANOVA $)$

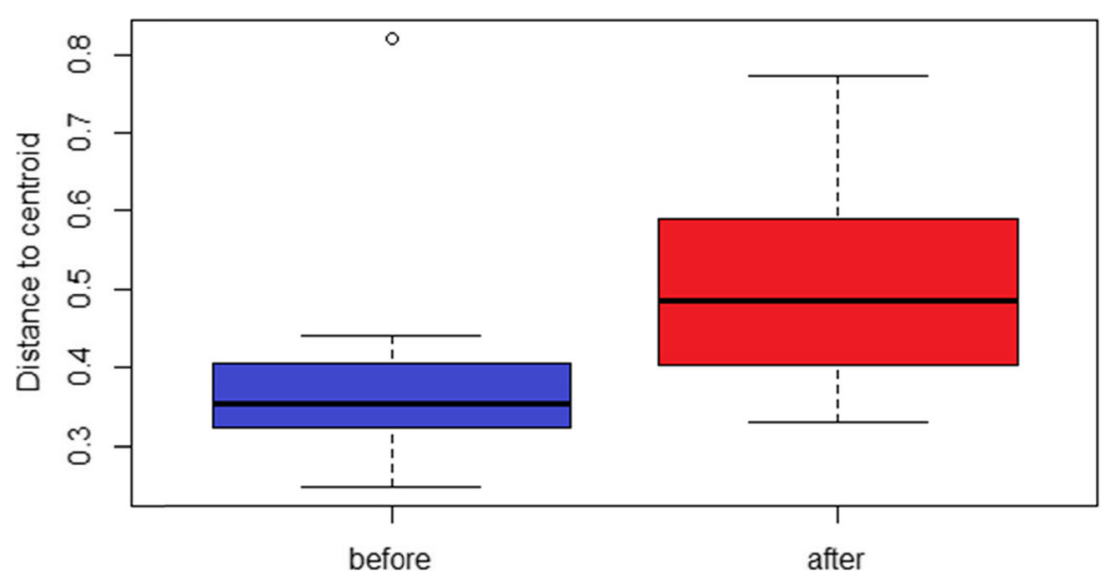

\section{Discussion}

We showed that ATB treatment with ceftiofur has a detectable impact on the GI microbiome of wild western lowland gorillas, mainly affecting the stability of the microbial community and the relative abundance of particular taxa. We also showed that the effect of ATB administration on diverse GI bacteria differed among individual hosts. This may be a reflection of the significant inter-individual variability observed in the GI microbiome of humans and non-human primates [30-33]. This baseline variation appears to be affected by continual intrinsic and extrinsic selective factors that vary among individuals (age, gender, and host genotype) or specific mutualistic interactions that stabilize community composition or diet [31, 34-36]. Moreover, based on our experience, ATB dosing is challenging in free-ranging animals and this variation may also be a factor. The dosage of ATBs is determined by an estimate of individual mass, which could result in a different dosage per kilogram in each individual. In addition, although it is always visually confirmed that administration of ATB injection was successful, there is a possibility that an individual did not received the full dose of treatment, such as when the injection is more subcutaneous than intramuscular or if some of the ATBs are not fully injected and might spray out the dart when it comes out of the gorilla. We acknowledge that the impact of ATB on GI microbiome of gorillas may also have been affected, to some extent, by sampling limitations and variations in sampling points. This may be the case of Mossoko, Mopambi, and Tembo. However, the fact that we observed steady, host-specific microbiome patterns in Makumba, Malui, and Mobangui (Fig. 5) implies that microbiome responses to ATB treatment in these individuals are still host dependent.

Gut microbiome diversity in samples of the silverback significantly decreased after ATB treatment. The same patterns were observed in other gorillas of different ages; however, we could not detect statistically significant differences, perhaps due to the smaller sample size. Therefore, we highlight both the challenges encountered when sampling wild apes and the difficulty of obtaining these kinds of samples during challenging situations such as a disease outbreak and administering of ATBs. When following groups of wild habituated lowland gorillas, the easiest individual to follow and to obtain distinguishable samples from is always the silverback male (due to the size and social role within the group), which explains why we had the highest number of samples from Makumba. Females and infants are much more difficult to sample, even under normal conditions. Thus, the results from the samples of these animals should be interpreted with caution. Nonetheless, the reduction of GI microbial diversity observed is concordant

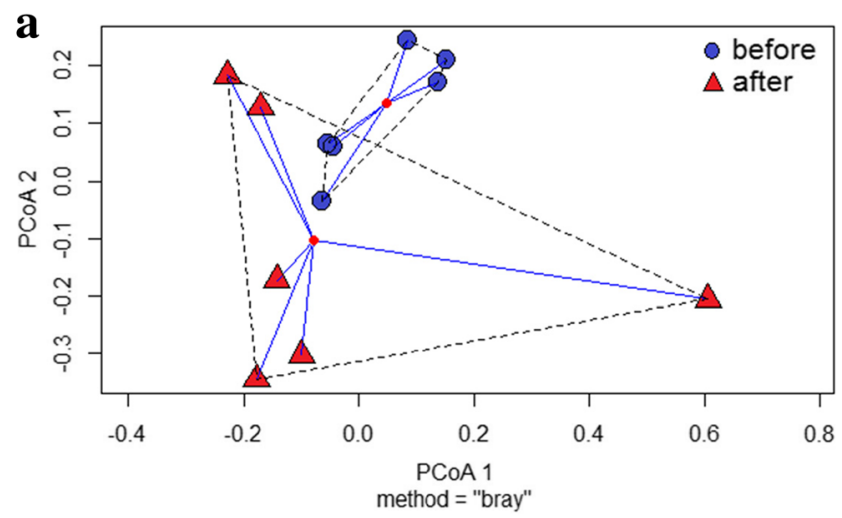

b

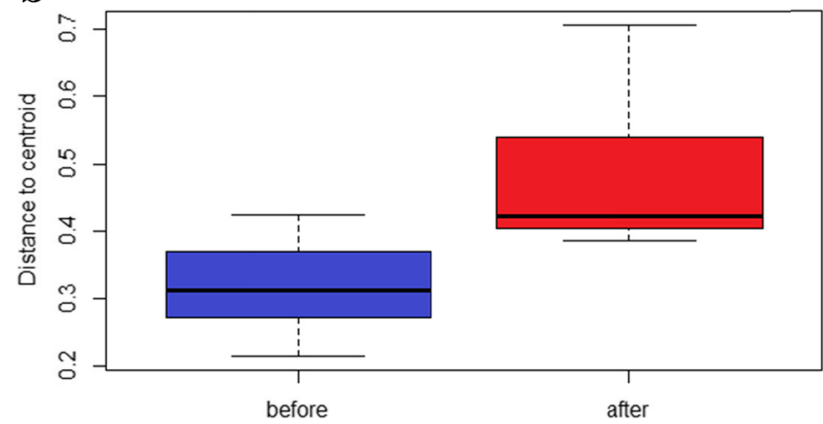

Fig. 3 GI microbiome dispersion of Makumba before and after ATB treatment (a) in multivariate space and (b) in boxplots $(p=0.002$, Wilcoxon's rank sum test) 

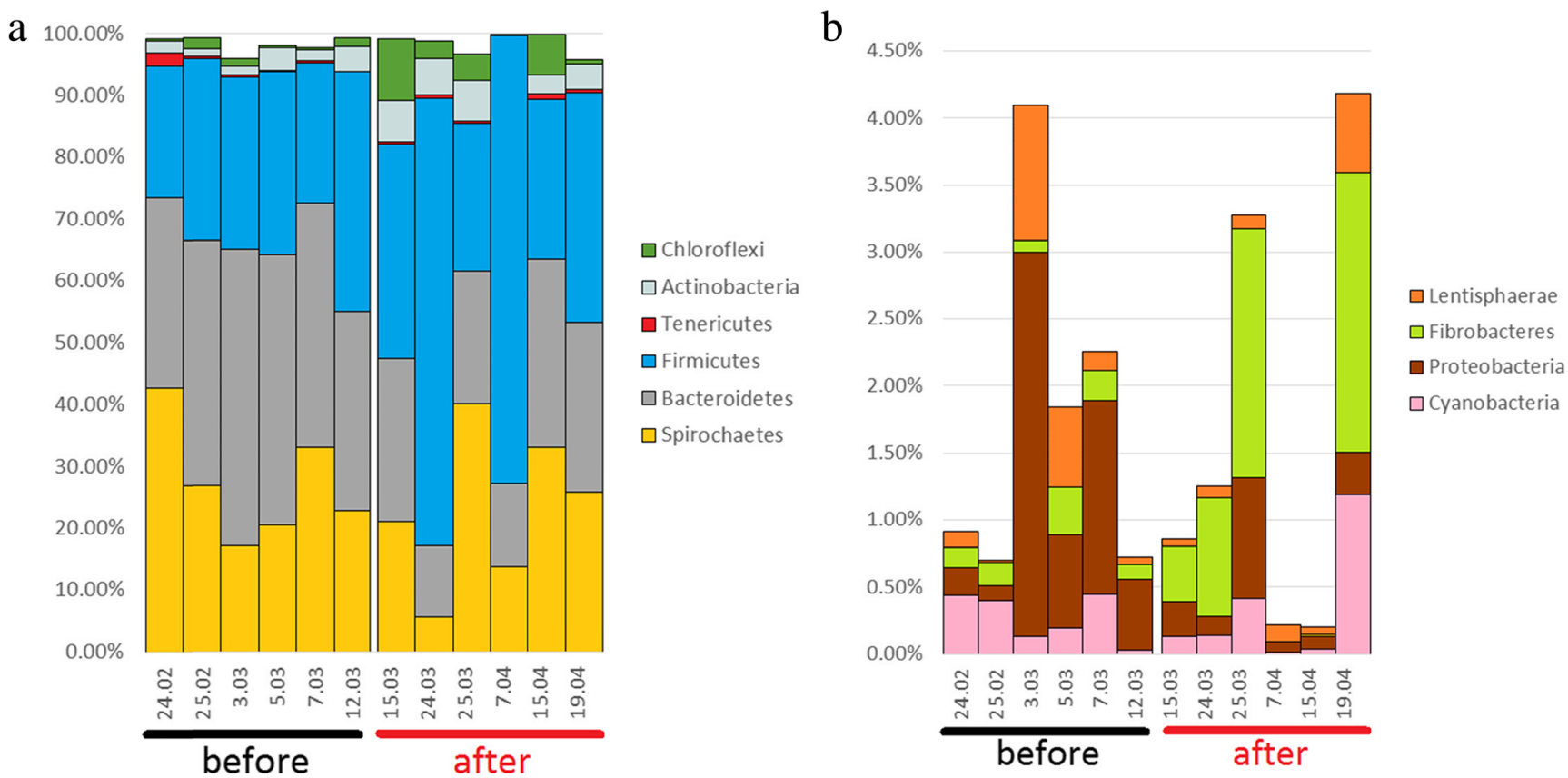

Fig. 4 Taxonomic profiles of major (a) and minor (b) phyla in samples before and after ATB treatment for the silverback Makumba

with other studies in humans and mice regardless of ATB type $[6-8,37]$. Decrease in microbial diversity may suggest that displacement of microorganisms sensitive to ATBs provides space for resistant strains to overgrow and dominate the colonic niche [7]. A collateral effect of this bacterial community turnover might have also been reflected by the less homogeneous and more dispersed microbiome observed in samples from all gorillas collected after ATB treatment.

The shifts in the levels of Firmicutes and Bacteroidetes, the two main phyla in the mammalian GI microbiome [31], are consistent with results of Welling et al. [3] and Pérez-Cobas et al. [6], who studied the impact of broad spectrum ATB in

Table 3 Indicator taxa with mean relative abundances in samples of Makumba before and after ATB treatment

\begin{tabular}{|c|c|c|c|c|c|}
\hline \multirow[t]{2}{*}{ Taxonomic rank } & \multirow[t]{2}{*}{ Indicator taxa before ATB } & \multirow[t]{2}{*}{ Indicator value } & \multirow[t]{2}{*}{ Probability } & \multicolumn{2}{|c|}{ Mean relative abundance \% (SD) } \\
\hline & & & & Before ATB & After ATB \\
\hline Phyla & Bacteroidetes & 0.64 & 0.004 & $38.96 * * *(6.61)$ & $21.75(7.86)$ \\
\hline \multirow[t]{4}{*}{ Family } & Unclassified (Mollicutes) & 0.96 & 0.016 & $0.31 * * *(0.67)$ & $0.01(0.03)$ \\
\hline & Unclassified (Deltaproteobacteria) & 0.83 & 0.017 & $0.03 * * *(0.06)$ & 0 \\
\hline & Bacteroidaceae & 0.83 & 0.024 & $0.78 * *(0.80)$ & $0.16(0.11)$ \\
\hline & Spirochaetaceae & 0.71 & 0.028 & $3.09 * * *(1.54)$ & $1.26(0.70)$ \\
\hline \multirow[t]{7}{*}{ Genus } & Unclassified (Mollicutes) & 0.96 & 0.01 & $0.31 * * *(0.67)$ & $0.01(0.03)$ \\
\hline & Bacteroides & 0.83 & 0.036 & $0.73 * *(0.81)$ & $0.15(0.10)$ \\
\hline & Unclassified (Deltaproteobacteria) & 0.83 & 0.015 & $0.03 * * *(0.06)$ & 0 \\
\hline & Ruminococcus & 0.78 & 0.035 & $0.42 * * *(0.31)$ & $0.12(0.08)$ \\
\hline & Mycoplasma & 0.74 & 0.039 & $0.09 * *(0.11)$ & $0.01(0.03)$ \\
\hline & Lachnobacterium & 0.74 & 0.025 & $0.37 * *(0.20)$ & $0.13(0.12)$ \\
\hline & Sphaerochaeta & 0.70 & 0.03 & $3.09 * * *(1.54)$ & $1.26(0.70)$ \\
\hline \multirow[t]{2}{*}{ Taxonomic rank } & \multirow[t]{2}{*}{ Indicator taxa after ATB } & \multirow[t]{2}{*}{ Indicator value } & \multirow[t]{2}{*}{ Probability } & \multicolumn{2}{|c|}{ Mean relative abundance \% (SD) } \\
\hline & & & & Before ATB & After ATB \\
\hline Family & Anaerolinaceae & 0.82 & 0.048 & $0.88(0.64)$ & $4.02 *(3.73)$ \\
\hline
\end{tabular}

Significant differences based on Wilcoxon's rank sum test, $* * * 0.01, * * 0.05$, and $* 0.1$ 


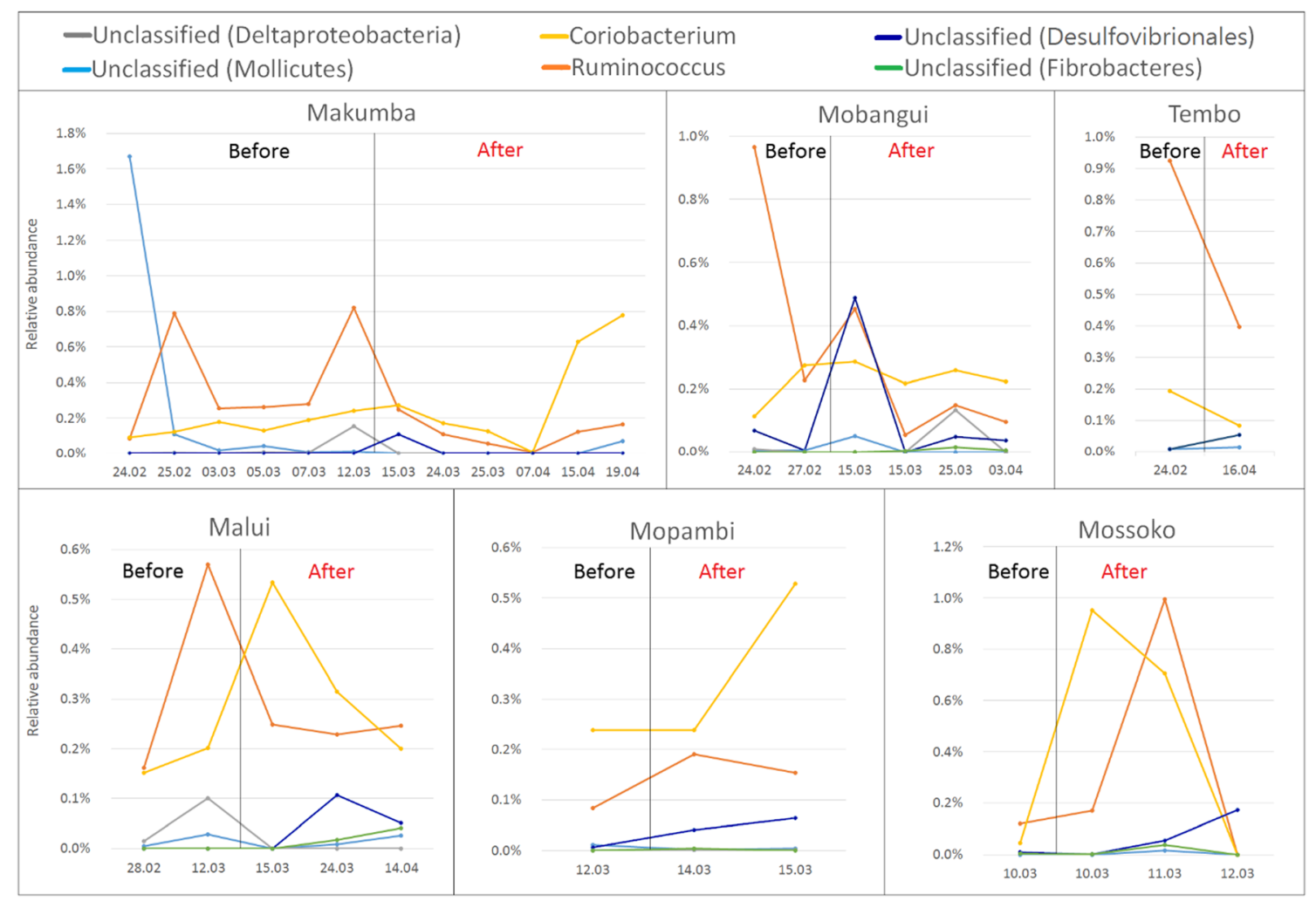

Fig. 5 Changes in relative abundances of six indicator taxa at the genus level during the sampling period in five studied gorillas. Plots are divided into two parts (black line), denoting samples collected before and after ATB treatment

humans. On the contrary, Panda et al. [7] detected a decrease of Firmicutes and an increase of Bacteroidetes after treatment with fluoroquinolones and B-lactams. Shifts in the ratios of these phyla have been associated with many physiological parameters, including obesity and an increased capacity to harvest energy from dietary sugars $[38,39]$. It is unclear whether the increased Firmicutes:Bacteroidetes ratios in gorillas after ATB treatment affect their energetic landscape. However, these shifts, as well as the emergence and decrease of other taxa, may reflect succession of particular bacterial community members and/or their metabolic functions after microbial taxa were affected by treatment.

Along with decreases in Bacteroidetes after ATB treatment, we observed an increase in relative abundance of Coriobacteriaceae, Chloroflexi (Anaerolinaceae), and Fibrobacteres. The Coriobacteriaceae family, also previously reported in chimpanzees [40] and humans [34], has been associated with energy metabolism, particularly with lipid turnover [36]. Chloroflexi has been detected in western lowland gorillas, eastern lowland gorillas, chimpanzees, bonobos [32], humans [41], and even ruminants [42]. This taxon is reported to contribute significantly to fermentative metabolism and increased organic matter conversion in other natural environments [43]. Fibrobacteres are important members of fibrolytic bacterial communities in mammalian guts, especially in cattle and pigs [44, 45], and are well known for their ability to degrade refractory plant structural polysaccharides [46]. Desulfovibrionales, which also increased after ATB treatment, has been reported in GI microbiome of ruminants, humans, and howler monkeys [42, 47, 48]. It has been demonstrated that members of Desulfovibrionales (e.g., Desulfovibrio, also detected in this study) have an important role in short-chain fatty acid (SCFA) turnover in the colon [49]. They may promote a shift in the metabolism of sugars, altering the synthesis by saccharolytic microorganisms of hydrolytic enzymes involved in carbohydrate breakdown [50].

A significant decrease in the relative abundance of Ruminococcus after ATB treatment was observed in all gorillas, with the exception of gorillas Mopambi and Mossoko, who were only sampled within a few days after treatment. Moreover, Mossoko was the only one receiving a second dose of ATB; however, we did not have available samples collected after the second treatment with EXCEDE $^{\circledR}$. Nevertheless, because of the similar characters of both ATBs (both are thirdgeneration cephalosporins), we expected similar effect on the GI microbiome. Contrary to our results, Looft et al. [13] reported that treatment with an antibiotic feed additive containing chlortetracycline, sulfamethazine, and penicillin caused increases in the relative abundance of Ruminococcus in the GI tract of swine. Ruminococcus spp. may be vital in cellulose breakdown in the gut ecosystem of hosts such as chimpanzees [40], mountain gorillas [51], humans [52], equines [53], and 
pigs [44]. Fibrolytic bacteria comprise part of a complex network in which different species play particular roles in fiber degradation. For example, both Ruminococcus and Fibrobacter are primary degraders of insoluble plant fiber, but degradation is performed differently, i.e., using different carbohydrate-processing modules by members of each genus [46].

It is likely that the emergence of specific taxa with fiber degradation and fermentative roles, such as Fibrobacter at the expense of Ruminococcus, taxa related to the Bacteroidaceae, and Deltaproteobacteria, may contribute to the absence of important nutrient-processing roles after some taxa have been affected after ATB treatment. Moreover, relative abundances of Lachnobacterium from the Lachnospiraceae family and Sphaerochaeta from the Spirochaetaceae family, the common members of human and animal GI microbiome [54-56], significantly decreased in samples collected after ATB treatment.

If the taxa with potential carbohydrate-processing roles dissipate and re-emerge after ATB treatment, then the GI microbiome of gorillas may be relatively resilient to extrinsic disturbances. This is a key consideration since fiber and carbohydrate processing and fermentation are critical functions in the GI tract of wild gorillas, especially when they rely heavily on fiber for nutrient and energy harvesting during low-fruit seasons, as was the case for this gorilla group during the study period [54, 57]. Indeed, disruption of the complex fibrolytic bacterial community at the GI level may lead to an inability to process high-fiber diets, with subsequent impact on the welfare of the host [52]. For instance, in equines, altered patterns of fiber degradation can result in illness and even death, caused by factors such as excessive accumulation of gas or decreased motility leading to intestinal impaction $[58,59]$.

Interestingly, we also recorded a significant decrease in the relative abundance of Mycoplasma in the silverback Makumba's samples collected after ATB treatment. Mycoplasma spp. are responsible for a variety of diseases, including contagious pleuropneumonia, in humans, domestic animals, wildlife, and non-human primates [60, 61]. Detected decreases in Mycoplasma levels, however, cannot be the direct result of the antibiotic treatment because cephalosporins act by inhibiting cell wall synthesis and are therefore ineffective against bacteria lacking a cell wall such as Mycoplasma.

Contrary to reports in humans [62-64], we did not detect any opportunistic pathogenic bacteria such as Clostridium, Klebsiella, or Staphylococcus, commonly linked with deleterious effects from ATB treatment or subsequent ATBassociated diarrhea. Moreover, no evident side effects were observed in treated gorillas (only gorilla Mossoko showed a runny stool once on the day of the second ATB treatment). This may suggest that the GI microbiome of wild apes may be less susceptible to ATBs compared to those of humans. However, as there have been no previous ATB treatments in the studied gorilla group and as the frequency of ATB administration is a key factor in boosting the emergence of potential pathogens and ATB-resistant bacteria, we suggest that additional studies should focus on apes more often treated with ATBs (e.g., mountain gorillas or captive great apes) [15, 16] or longer-term studies in lowland gorillas with more frequent sampling. However, this scenario is hardly attainable with wild lowland gorillas due to the rare administration of ATB treatment, dispersed groups, and challenging field/ research conditions.

Although interpretations of results based on comparisons with untreated animals are limited due to the low sample size from untreated individuals, we were able to confirm the effect of ATB treatment on the microbial composition when comparing the silverback Makumba and blackback Kunga (Fig. S6). In this regard, the fact that trends in the levels of all indicator taxa were similar for both males in samples collected before ATB treatment, but not after, is not only support for the effect of ATB on the gut microbiome of wild apes but also support for successful administration of ATB treatment. This is also supported by the fact that all animals successfully recovered from the outbreak. Nonetheless, to further validate the influence of ATBs on the gut microbiome of great apes and a possible resilience or recovery, more controlled studies with increased sample sizes are needed.

\section{Conclusions}

Despite the challenging sampling conditions and limitations covering the whole treatment period in all studied gorillas (including those not receiving treatment), our study is the first to describe how ATB treatment affects GI bacterial communities in wild apes. In summary, our results showed significant changes in the GI microbial composition caused by ATB treatment with differences dependent on individual host and potential resilience of the gorilla GI microbiome in terms of metabolic capacity. The consequences of the changes may not be immediately clear; however, as shown in the human GI microbiome, ATBs do appear to have long-term impacts [4]. Although no serious health problems were observed after ATB treatment in the studied gorillas and all individuals recovered fully from the outbreak, further research is warranted on the pervasive and persisting effect of ATBs in wild apes after ATB administration, including the presence and spread of ATB-resistant strains. Additionally, future studies should assess whether the effect of ATB treatment described in this study may be generalized to other ATB types. These data provide important conservation implications for the considerations of ATB treatment in wild animals. These results are suggestive and point to the need for more controlled sampling. Until then, we urge caution in wild ape ATB treatments and stress the need for proper risk assessment and further study. 
Acknowledgments We express our gratitude to the Government of the Central African Republic and to the World Wildlife Fund for granting permission to conduct our research in the Central African Republic, the Primate Habituation Programme (Dzanga-Ndoki National Park, DzangaSangha Protected Areas) for the logistical support in the field, and all local trackers and assistants from Bai Hokou for their help with sample collection. Moreover, we would like to thank to Zoo Liberec for the financial support during ATB treatment of gorillas. We thank to the Roy J. Carver Biotechnology Center, High-Throughput Sequencing and Genotyping Unit, University of Illinois at Urbana-Champaign for providing the amplicon sequencing using Illumina MiSeq Platform. This publication derives from the HPI-Lab, Laboratory for Infectious Diseases Common to Humans and (Non-Human) Primates, Czech Republic. This work was financially supported by the Internal Grant Agency of University of Veterinary and Pharmaceutical Sciences Brno 47/2013/FVL, by the institutional support of the Institute of Vertebrate Biology, Academy of Sciences of the Czech Republic (RVO 68081766), and co-financed from European Social Fund and State Budget of the Czech Republic (project OPVK CZ.1.07/2.3.00/20.0300). Sequencing was supported by the US National Science Foundation (NSF) 0935347 and the University of Illinois at Urbana-Champaign.

Compliance with Ethical Standards The non-invasive sample collection was conducted according to the Convention on Human Rights and Biomedicine of the Council of Europe and Directive 86/609/EEC on the Protection of Animals Used for Experimental and Other Scientific Purposes and meet the International Guiding Principles for Biomedical Research Involving Animals, as issued by the Council for International Organizations of Medical Sciences (C.I.O.M.S., c/o WHO, CH 1211 Geneva 27, Switzerland). The research adhered to the legal requirements of the Central African Republic. Importation of the samples to the EU was approved by the State Veterinary Authority of the Czech Republic. The sequencing was conducted under University of Illinois IACUC protocol 11046.

Conflict of Interest The authors declare that they have no conflict of interests.

\section{References}

1. Berg R (1996) The indigenous gastrointestinal microflora. Trends Microbiol 4:430-435

2. Isolauri E, Salminen S, Ouwehand AC (2004) Probiotics. Best practice and research. Clin Gastroenterol 18:299-313

3. Welling GW, Meijer-Severs GJ, Helmus G, van Santen E, Tonk RHJ, de Vries-Hospers HG, van der Waaij D (1991) The effect of ceftriaxone on the anaerobic bacterial flora and the bacterial enzymatic activity in the intestinal tract. Infection 19:313-316

4. Jernberg C, Löfmark S, Edlund C, Jansson JK (2007) Long-term ecological impacts of antibiotic administration on the human intestinal microbiota. ISME J 1:56-66

5. Yap IKS, Li JV, Saric J, Martin F-P, Davies H, Wang Y, Wilson ID, Nicholson JK, Utzinger J, Marchesi JR, Holmes E (2008) Metabonomic and microbiological analysis of the dynamic effect of vancomycin-induced gut microbiota modification in the mouse. $\mathrm{J}$ Proteome Res 7:3718-3728

6. Pérez-Cobas AE, Gosalbes MJ, Friedrichs A, Knecht H, Artacho A, Eismann K, Otto W, Rojo D, Bargiela R, von Bergen M, Neulinger SC, Däumer C, Heinsen FA, Latorre A, Barbas C, Seifert J, dos Santos VM, Ott SJ, Ferrer M, Moya A (2013) Gut microbiota disturbance during antibiotic therapy: a multi-omic approach. Gut 62:1591-1601
7. Panda S, El khader I, Casellas F, Vivancos JL, Cors MG, Santiago A, Cuenca S, Guarner F, Manichanh C (2014) Short-term effect of antibiotics on human gut microbiota. PLoS One 9, e95476

8. Raymond F, Ouameur AA, Déraspe M, Iqbal N, Gingras H, Dridi B, Leprohon P, Plante P-L, Giroux R, Ėve B, Frenette J, Boudreau DK, Simard J-L, Chabot I, Domingo M-C, Trottier S, Boissinot M, Huletsky A, Roy PH, Ouellette M, Bergeron MG, Corbeil J (2015) The initial state of the human gut microbiome determines its reshaping by antibiotics ISME J. 1-14

9. Willing BP, Russell SL, Finlay BB (2011) Shifting the balance: antibiotic effects on host microbiota mutualism. Nat Rev Microbiol 9:233-243

10. Carlet J (2012) The gut is the epicentre of antibiotic resistance. Antimicrob Resist Infect Control 1:39

11. Janatova M, Albrechtova K, Petrzelkova KJ, Dolejska M, Papousek I, Masarikova M, Cizek A, Todd A, Shutt K, Kalousova B, Profousova-Psenkova I, Modry D, Literak I (2014) Antimicrobialresistant Enterobacteriaceae from humans and wildlife in DzangaSangha Protected Area, Central African Republic. Vet Microbiol 171:422-431

12. Dethlefsen L, Huse S, Sogin ML, Relman DA (2008) The pervasive effects of an antibiotic on the human gut microbiota, as revealed by deep 16S rRNA sequencing. PLoS Biol 6, e280

13. Looft T, Johnson TA, Allen HK, Bayles DO, Alt DP, Stedtfeld RD, Sul WJ, Stedtfeld TM, Chai B, Cole JR, Hashsham SA, Tiedje JM, Stanton TB (2012) In-feed antibiotic effects on the swine intestinal microbiome. Proc Natl Acad Sci U S A 109:1691-1696

14. Zhao Y, Wu J, Li JV, Zhou N-Y, Tang H, Wang Y (2013) Gut microbiota composition modifies fecal metabolic profiles in mice. J Proteome Res 12:2987-2999

15. MGVP and WCS (2008) Conservation medicine for gorilla conservation. In: Stoinski TS, Steklis HD, Mehlman PT (eds) Conservation in the 21st Century: Gorillas as a Case Study. Springer Science + Business Media, LLC, pp 57-78

16. Spelman LH, Gilardi KV, Lukasik-Braum M, Kinani JF, Nyirakaragire E, Lowenstine LJ, Cranfield MR (2013) Respiratory disease in mountain gorillas (Gorilla beringei beringei) in Rwanda, 1990-2010: outbreaks, clinical course, and medical management. J Zoo Wildl Med 44:1027-1035

17. Szentiks CA, Köndgen S, Silinski S, Speck S, Leendertz FH (2009) Lethal pneumonia in a captive juvenile chimpanzee (Pan troglodytes) due to human-transmitted human respiratory syncytial virus (HRSV) and infection with Streptococcus pneumoniae. J Med Primatol 38:236-240

18. Prescott JF (2006) Beta-lactam antibiotics: cephalosporins. In: Giguere S, Prescott JF, Baggot JD, Walker RD, Dowling PM (eds) Antimicrobial therapy in veterinary medicine, 4th edn. Blackwell Publishing, Ames, Iowa, pp 139-158

19. Crane JP, Bryson WL, Anderson YC, Callahan JK, Portis ES, Lindeman CJ, Lucas MJ, Robb EJ (2006) Duration of efficacy of ceftiofur crystalline free acid sterile suspension against clinical disease in grower pigs challenged with Actinobacillus pleuropneumoniae. J Swine Health Prod 14:302-306

20. Collard WT, Cox SR, Lesman SP, Grover GS, Boucher JF, Hallberg JW, Robinson JA, Brown SA (2011) Pharmacokinetics of ceftiofur crystalline-free acid sterile suspension in the equine. $\mathrm{J}$ Vet Pharmacol Ther 34:476-481

21. Adkesson MJ, Junge RE, Allender MC, Martín-Jiménez T (2012) Pharmacokinetics of a long-acting ceftiofur crystalline-free acid formulation in Asian elephants (Elephas maximus). Am J Vet Res 73:1512-1518

22. Meegan J, Collard WT, Grover GS, Pussini N, Bonn WGV, Gulland FMD (2013) Pharmacokinetics of ceftiofur crystalline-free acid (EXCEDE sterile suspension) administered via intramuscular injection in wild California sea lions (Zalophus californianus). J Zoo Wildl Med 44:714-720 
23. Jeraldo P, Kalari K, Chen X, Bhavsar J, Mangalam A, White B, Nelson H, Kocher JP, Chia N (2014) IM-TORNADO: a tool for comparison of $16 \mathrm{~S}$ reads from paired-end libraries. PLoS One 9, e114804

24. Clarke K, Gorley R (2006) PRIMER v6: user manual/tutorial. PRIMER-E, Plymouth

25. Caporaso JG, Kuczynski J, Stombaugh J, Bittinger K, Bushman FD, Costello EK, Fierer N, Peña AG, Goodrich JK, Gordon JI, Huttley GA, Kelley ST, Knights D, Koenig JE, Ley RE, Lozupone CA, McDonald D, Muegge BD, Pirrung M, Reeder J, Sevinsky JR, Turnbaugh PJ, Walters WA, Widmann J, Yatsunenko T, Zaneveld J, Knight R (2010) QIIME allows analysis of highthroughput community sequencing data. Nat Methods 7:335-336

26. Dufrêne M, Legendre P (1997) Species assemblages and indicator species: the need for a flexible asymmetrical approach. Ecol Monogr 67:345-366

27. Roberts DW (2013) labdsv: ordination and multivariate analysis for ecology. R package version 1.6-1

28. R Development Core Team (2008) $R$ : a language and environment for statistical computing. R Foundation for Statistical Computing, Vienna, Austria. ISBN 3-900051-07-0

29. Oksanen J, Blanchet FG, Kindt R, Legendre P, Minchin PR, O'Hara RB, Simpson GL, Solymos P, Stevens MHH, Wagner H (2013) Vegan: community ecology package. $\mathrm{R}$ package version 2.0-10

30. McKenna P, Hoffmann C, Minkah N, Aye P, Lackner A, Liu Z, Lozupone CA, Hamady M, Knight R, Bushman F (2008) The macaque gut microbiome in health, lentiviral infection, and chronic enterocolitis. PLoS Pathog 4:e20

31. Mariat D, Firmesse O, Levenez F, Guimarăes VD, Sokol H, Doré J, Corthier G, Furet JP (2009) The Firmicutes/Bacteroidetes ratio of the human microbiota changes with age. BMC Microbiol 9:123128

32. Ochman H, Worobey M, Kuo CH, Ndjango JBN, Peeters M, Hahn BH, Hugenholtz P (2010) Evolutionary relationships of wild hominids recapitulated by gut microbial communities. PLoS Biol 8 , e1000546

33. Szekely B, Singh J, Marsh T, Hagedorn C, Werre S, Kaur T (2010) Fecal bacterial diversity of human-habituated wild chimpanzees (Pan troglodytes schweinfurthii) at Mahale Mountains National Park, Western Tanzania. Am J Primatol 72:566-574

34. Harmsen HJM, Wildeboer-Veloo ACM, Grijpstra J, Knol J, Degener JE, Welling GW (2000) Development of 16S rRNAbased probes for the Coriobacterium group and the Atopobium cluster and their application for enumeration of Coriobacteriaceae in human feces from volunteers of different age groups. Appl Environ Microbiol 66:4523-4527

35. Zoetendal EG, Akkermans ADL, van Vliet WMA, de Visser JGM, de Vos WM (2001) The host genotype affects the bacterial community in the human gastrointestinal tract. Microb Ecol Health D 13: 129-134

36. Turnbaugh PJ, Hamady M, Yatsunenko T, Cantarel BL, Duncan A, Ley RE, Sogin ML, Jones WJ, Roe BA, Affourtit JP, Egholm M, Henrissat B, Heath AC, Knight R, Gordon JI (2009) A core gut microbiome in obese and lean twins. Nature 457:480-484

37. Antonopoulos DA, Huse SM, Morrison HG, Schmidt TM, Sogin ML, Young VB (2009) Reproducible community dynamics of the gastrointestinal microbiota following antibiotic perturbation. Infect Immun 77:2367-2375

38. Ley RE, Turnbaugh PJ, Klein S, Gordon JI (2006) Microbial ecology: human gut microbes associated with obesity. Nature 444 : 1022-1023

39. Turnbaugh PJ, Backhed F, Fulton L, Gordon JI (2008) Marked alternations in the distal gut microbiome linked to diet-induced obesity. Cell Host Microbe 3:213-223
40. Moeller AH, Degnan PH, Pusey AE, Wilson ML, Hahn BH, Ochman H (2012) Chimpanzees and humans harbour compositionally similar gut enterotypes. Nature 3:1179

41. Davenport ER, Mizrahi-Man O, Michelini K, Barreiro LB, Ober C, Gilad Y (2014) Seasonal variation in human gut microbiome composition. PLoS One 9, e90731

42. Omoniyi L, Jewell K, Isah O, Neumann A, Onwuka C, Onagbesan O, Suen G (2013) An analysis of the ruminal bacterial microbiota in West African Dwarf sheep fed grass- and tree-based diets. J Appl Microbiol 11:1094-1105

43. Yamada T, Sekiguchi Y, Hanada S, Imachi H, Ohashi A, Harada H, Kamagata Y (2006) Anaerolinea thermolimosa sp. nov., Levilinea saccharolytica gen. nov., sp. nov. and Leptolinea tardivitalis gen. nov., sp. nov., novel filamentous anaerobes, and description of the new classes Anaerolineae classis nov. and Caldilineae classis nov. in the bacterial phylum Chloroflexi. Int J Syst Evol Microbiol 56: $1331-1340$

44. Varel VH, Yen JT (1997) Microbial perspective on fiber utilization by swine. J Anim Sci 75:2715-2722

45. Qi M, Nelson KE, Daugherty SC, Nelson WC, Hance IR, Morrison M, Forsberg CW (2005) Novel molecular features of the fibrolytic intestinal bacterium Fibrobacter intestinalis not shared with Fibrobacter succinogenes as determined by suppressive subtractive hybridization. J Bacteriol 187:3739-3751

46. Flint HJ, Bayer EA, Rincon MT, Lamed R, White BA (2008) Polysaccharide utilization by gut bacteria: potential for new insights from genomic analysis. Nat Rev Microbiol 6:121-131

47. Ley RE, Hamady M, Lozupone C, Turnbaugh PJ, Ramey RR, Bircher JS, Schlegel ML, Tucker TA, Schrenzel MD, Knight R, Gordon JI (2008) Evolution of mammals and their gut microbes. Science 320:1647-1651

48. Nakamura N, Amato KR, Garber P, Estrada A, Mackie RI, Gaskins HR (2011) Analysis of the hydrogenotrophic microbiota of wild and captive black howler monkeys (Alouatta pigra) in Palenque National Park, Mexico. Am J Primatol 73:909-919

49. Gibson G, Macfarlane S, Macfarlane G (1993) Metabolic interactions involving sulphate-reducing and methanogenic bacteria in the human large intestine. FEMS Microbiol Ecol 12:117-125

50. Newton D, Cummings J, Macfarlane S, Macfarlane G (1998) Growth of a human intestinal Desulfovibrio desulfuricans in continuous cultures containing defined populations of saccharolytic and amino acid fermenting bacteria. J Appl Microbiol 85:372-380

51. Frey JC, Rothman JM, Pell AN, Nizeyi JB, Cranfield MR, Angert ER (2006) Fecal bacterial diversity in a wild gorilla. Appl Environ Microbiol 72:3788-3792

52. Bernalier-Donadille A (2010) Fermentative metabolism by the human gut microbiota. Gastroentrol Clin Biol 34:S16-S22

53. Julliand V, de Vaux A, Millet L, Fonty G (1999) Identification of Ruminococcus flavefaciens as the predominant cellulolytic bacterial species of the equine cecum. Appl Environ Microbiol 60:37383741

54. Gomez A, Petrzelkova K, Yeoman CJ, Vlckova K, Mrazek J, Koppova I, Carbonero F, Ulanov A, Modry D, Todd A, Torralba M, Nelson KE, Gaskins HR, Wilson B, Stumpf RM, White BA, Leigh SR (2015) Gut microbiome composition and metabolomic profiles of wild western lowland gorillas (Gorilla gorilla gorilla) reflect host ecology. Mol Ecol 24:2551-2565

55. Moeller AH, Li Y, Ngole EM, Ahuka-Mundeke S, Lonsdorf EV, Pusey AE, Peeters M, Hahn BH, Ochman H (2014) Rapid changes in the gut microbiome during human evolution. Proc Natl Acad Sci U S A 111:16431-1635

56. Pajarillo EAB, Chae JP, Balolong MP, Kim HB, Seo K-S, Kang DK (2014) Pyrosequencing based analysis of fecal microbial communities in three purebred pig lines. J Microbiol 52:646-651

57. Doran-Sheehy D, Mongo P, Lodwick J, Conklin-Brittain N (2009) Male and female western gorilla diet: preferred foods, use of 
fallback resources, and implications for ape versus old world monkey foraging strategies. Am J Phys Anthropol 140:727-738

58. Argenzio R (1975) Functions of the equine large intestine and their interrelationship in disease. Cornell Vet 65:303-330

59. Boyd L, Houpt KA (1994) Przewalski's horse: the history and biology of an endangered species. SUNY Press

60. Waites KB, Talkington DF (2004) Mycoplasma pneumoniae and its role as a human pathogen. Clin Microbiol Rev 17:697-728

61. Sumithra TG, Chaturvedi VK, Susan C, Siju SJ, Rai AK, Harish C, Sunita SC (2013) Mycoplasmosis in wildlife: a review. Eur J Wildl Res 59:769-781

62. Högenauer C, Langner C, Beubler E, Lippe IT, Schicho R, Gorkiewicz G, Krause R, Gerstgrasser N, Krejs GJ, Hinterleitner
TA (2006) Klebsiella oxytoca as a causative organism of antibiotic-associated hemorrhagic colitis. N Engl J Med $355: 2418-2426$

63. Theriot CM, Young VB (2013) Microbial and metabolic interactions between the gastrointestinal tract and Clostridium difficile infection. Landes Biosci 5:73-82

64. Baba-Moussa L, Ahissou H, Azokpota P, Assogba B, Anagonou MMAS, Keller D, Sanni A, Prévost G (2010) Toxins and adhesion factors associated with Staphylococcus aureus strains isolated from diarrhoeal patients in Benin. Afr J Biotechnol 9:604-611 